\section{Características del suelo en los cultivos de cafés especiales del municipio de Calarcá - Colombia}

\section{Soil characteristics in specialty coffee crops in the municipality of Calarca - Colombia}

\author{
Víctor Cerón-Chacón*, Juan Ricardo Gómez-Duque*, Luis Miguel Mejía- \\ Giraldo**, Laura Sofia Torres-Valenzuela**, Adriana Lucía Andrade***, Deisy \\ Natalia Hormiga-García***
}

\begin{abstract}
* Docente investigador, Sena Agroindustrial seccional Quindío, vhceron8@misena.edu.co
* Docente investigador, Sena Agroindustrial seccional Quindío, jrgomezd@misena.edu.co

** Docente investigador, Universidad La Gran Colombia seccional Armenia, mejiagluismiguel@miugca. edu.co

** Docente investigadora, Universidad La Gran Colombia seccional Armenia, torresvallaura@miugca.edu.

*** Auxiliar de investigación, Sena Agroindustrial seccional Quindío, adriana.a2009@hotmail.com

*** Auxiliar de investigación, Sena Agroindustrial seccional Quindío, dnhormiga@misena.edu.co
\end{abstract}

\section{Resumen}

La producción de café es un renglón económico de importancia significativa en Colombia que cobra cada vez más relevancia por la declaratoria de la Unesco de Paisaje Cultural Cafetero Colombiano, generando oportunidades para el sector productivo, pero también implica nuevos retos asociados a la calidad del café que le permita acceder a los mercados especializados, destacando la dinámica económica creciente de los cafés especiales. Existe una relación entre las prácticas agronómicas -incluyendo el origen y características del suelo- y la respuesta de la bebida en taza y la perfilación con relación a la expresión de los cafés especiales definidos por la SCAA (Specialty Coffee Assotiation of America). Por lo anterior, en este proyecto se realizó una clasificación de los suelos de la región de Calarcá ( $\mathrm{pH}$, materia orgánica, elementos mayores, clase textural, hierro y aluminio). A partir de análisis multivariado se encontraron dos tipologías de suelo determinados principalmente por el $\mathrm{pH}$, concentración de calcio y los niveles de aluminio del suelo, así como el uso de semisombra, siendo parámetros relevantes para dicho tipo de café.

Palabras clave: Catación, clústeres, correspondencias múltiples, ecotopo, perfilación.

\begin{abstract}
Coffee production is an economic sector of significant importance in Colombia, which is becoming increasingly important for the declaration of Unesco "Colombian Coffee Cultural Landscape" generating opportunities for the productive sector, but it also involves new challenges associated with quality Coffee order to gain access to specialized markets, highlighting the growing economic dynamics of specialty coffees. There is a relationship between agronomic practices - including the origin and characteristics of the soil - and the response of the drink cup and profiling relative to the expression of the specialty coffees defined by the SCAA (Specialty Coffee assotiation of America). Therefore, in this project a classification of soils in the region of Calarcá ( $\mathrm{pH}$, organic matter, major elements, textural class, iron and aluminum) was performed. From multivariate analysis, two types of soil mainly determined by the $\mathrm{pH}$, concentration of calcium and aluminum levels of soil and the use of partial shade, being relevant to that type of coffee parameters were found.
\end{abstract}

Keywords: Clusters, cupping, ecotope, multiple correspondance, profiling, SCAA.
Recibido: $20 / 02 / 2015$

Revisado: $16 / 03 / 2015$

Aceptado: 10/12/2015

Correspondencia de autor:

mejiagluismiguel@miugca. edu.co

(C) 2015 Universidad La Gran Colombia. Este es un artículo de acceso abierto, distribuido bajo los términos de la licencia Creative Commons Attribution License, que permite el uso ilimitado, distribución y reproducción en cualquier medio, siempre que el autor original y la fuente se acrediten.

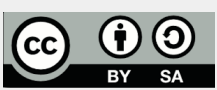

\section{Cómo citar:}

Cerón-Chacón, V., Gómez-Duque, J.R., Mejía-Giraldo, L.M., Torres-Valenzuela, L., Andrade, A., \& Hormiga-García, D.(2015) Caracteristicas del suelo en los cultivos de cafés especiales del municipio de Calarcá-Colombia. UGCiencia 21, 29-38. 


\section{Introducción}

El café es uno de los principales bienes básicos que se transan en los mercados financieros más importantes de la economía mundial y del comercio internacional. Este es un producto crucial para el crecimiento económico y la estabilidad política de alrededor de 50 países en desarrollo, dado que es producido por 17 a 20 millones de familias y genera ingresos de más de 15 billones de dólares anuales. Además, en Colombia, la producción cafetera otorga ingresos a 516000 familias, de las cuales el $95 \%$ tienen predios de menos de 5 hectáreas; esta actividad genera alrededor de 700000 empleos directos y proporciona un millón y medio de manera indirecta (Lozano y Yoshida, 2008).

El Quindío sigue siendo el mayor generador de ingresos a las familias rurales y contribuye en buena parte con el producto interno bruto (PIB) del departamento. Hoy se estiman alrededor de 5600 productores de café, de las cuales 4000 son pequeños y medianos cafeteros, que dependen del grano de café, pero se deben dar cambios importantes en cuanto a la producción y manejo de la calidad basado en la producción, proceso de beneficio húmedo y seco, para competir en el mercado de cafés especiales, los cuales son diferenciados por sus características organolépticas, condiciones de producción, entre otros aspectos que los hace diferentes del café que se comercializa normalmente en los mercados nacionales e internacionales, donde obtengan un mejor ingreso que supere los costos de producción por arroba de café pergamino seco, dado que bajo esta condición del grano se basa su respectiva comercialización.

El café es uno de los productos más comercializados y es una de las bebidas más populares en el mundo por su sabor así como otras características sensoriales. Además, los granos de café son una importante fuente de algunos principios activos entre los que se encuentran la cafeína y los ácidos clorogénicos. Este tipo de compuestos son utilizados como materia prima en las industrias farmacéutica, cosmética y alimentaria.

Por otra parte, hay una alta tendencia por el consumo de cafés con connotación especial, asociados a diversas dinámicas de mercado que generan mayores precios de comercialización, lo cual permitiría impactar el eslabón de producción primaria (productores) con mayor rentabilidad asociada a precios de venta mayores.
Dadas las condiciones anteriores, es importante establecer las relaciones existentes entre las características del suelo, el proceso productivo, la calidad de taza y la perfilación de acuerdo a lo establecido por la Asociación Americana de Cafés Especiales (SCAA por su nombre en inglés). Esto permitiría establecer el clúster e identificar aspectos relacionados con la producción cafetera de origen de esta región, en busca de generar valor agregado y fortalecimiento de las cadenas productivas que contribuyan a la sostenibilidad de los productores cafeteros, debido al necesario abordaje del tema de la sostenibilidad en la agricultura con el fin de centrarse en aspectos asociados al abordaje y análisis del medio ambiente y los aspectos técnicos, e impactando, en última instancia, sobre todo los aspectos sociales de la misma (Binder et al., 2009).

Con base en lo anterior y con el fin de detectar variables fundamentales de producción de café con respecto al recurso suelo y su manejo agronómico, se plantea un sistema de análisis estadístico soportado en el análisis multivariado, el que implica el examen de factores que debidamente correlacionados, explican los diferentes aspectos evaluados de manera conjunta, y permiten establecer potenciales indicadores, así como conglomerados (clústeres) de productores según las variables que ejerzan mayor peso estadístico con respecto a la variable indicativa de la calidad de taza como tal.

El abordaje del concepto de caracterización así como el diseño de indicadores, son aspectos fundamentales para el análisis de la trazabilidad en diferentes contextos siempre y cuando sean significativos con base en métodos de evaluación coherentes, resaltándose para el ámbito agrícola que los aspectos medioambientales, técnicos de producción, manejo de poscosecha, entre otros, pueden afectar la productividad en agroecosistemas específicos por motivo de la sostenibilidad en dicho renglón productivo, ya que dada la multifuncionalidad e integración holística de la agricultura y la aplicabilidad de los resultados (Binder et al., 2009).

En respuesta a las deficiencias de estos indicadores, Binder et al. (2009) afirman que se requiere la integración de métodos de evaluación para el sector agrícola con el fin de una sostenibilidad posible; sin embargo, se debe tener en cuenta que un indicador determinado no dice nada acerca de la sostenibilidad, a menos que dé un valor de referencia que brinde criterios para la definición de umbrales (Lancker y Nijkamp, 2000). Sumado a lo 
anterior, Lundin (2003), y Berke y Manta (1999), afirman que los índices y métodos estadísticos enfocados a la agricultura se pueden utilizar para anticipar y evaluar las condiciones y tendencias sobre el nivel de aceptación o rechazo de las comunidades con respecto a políticas sostenibles, proporcionar información de alerta temprana para prevenir daños económicos, sociales y ambientales, formular estrategias y comunicar ideas y apoyar la toma de decisiones.

Con respecto a la selección y desarrollo de índices para la agricultura dentro de un contexto de desarrollo sostenible, se pueden distinguir dos enfoques, como lo resalta Lundin (2003) lo cuales son el enfoque del marco y el conjunto de los índices e indicadores de desarrollo sostenible de parte de los expertos e investigadores; mientras el segundo se soporta en la participación de los diferentes actores o partes interesadas en el diseño de la estructura y el proceso de selección de los índices de desarrollo sostenible. Autores como Holmberg y Karlsson (1992) introdujeron el concepto de indicadores socio-ecológicos los cuales giran en torno al análisis correlacional entre condiciones sociales en asocio con el entorno ecológico de manera integral y holística y dada la necesidad de analizar de manera simultáneamente el medio ambiente y los componentes sociales del desarrollo sostenible en la agricultura, se han establecido parámetros para medición de la sostenibilidad como aspecto determinante (Prescott-Allen, 1995; Wackernagel y Rees, 1996), los cuales se componen de la valoración de los ecosistemas y el bienestar del ser humano; pero también a través de indicadores que sean relevantes y significativos para los negocios, lo cual es afín a lo mencionado por Rajesh et al (2008) y corroborado por Epstein (2009). No obstante, existen evidencias que explican el por qué las empresas actúan en conjunción con una verdadera responsabilidad social (Bansal y Roth, 2000; Schmidt-Bleek, 1994), donde se debe integrar la productividad con aspectos ambientales, tal como lo evidencian Wood (1991); Ebert y Welsch (2004). Es de agregar que el desarrollo de modelos que se centran en una mejor comprensión de las relaciones causales como lo aseveran Epstein y Westbrook (2001), los cuales resaltan los vínculos dentro de las organizaciones y las herramientas que personas que toman decisiones pueden tener para mejorar tanto la relación con los demás actores "stakeholders" sin ir en contraposición con la rentabilidad económica y mejorar el rendimiento de las organizaciones, aspecto que es corroborado por Freeman (1984), quién resalta la importancia de la identificación de los temas sociales y ambientales asociados con sectores específicos y la ubicación geográfica de los mismos, para lo cual Ness et al (2007) plantean una clasificación y evaluación de los indicadores, que se puede hacer sobre la base de dimensiones generales de la medición como el aspecto de sostenibilidad a abordar, las técnicas y métodos para su medición y análisis, la dinámica correlacional entre los diferentes aspectos que atañen a ambientes sostenibles y su adaptabilidad a diferentes condiciones, como es en el caso del sector agrícola.

Para el caso específico de café, el sabor y aroma característicos son resultado de una combinación de cientos de compuestos químicos que se desarrollan durante el crecimiento del fruto y promovidos durante el proceso de tostión. Este proceso se puede dividir en tres etapas consecutivas que son el secado del grano, la calcinación o pirolisis y el enfriamiento. La primera etapa se caracteriza por una liberación lenta de agua y sustancias volátiles, durante el primer medio de procesamiento, donde el grano cambia de color verde a amarillo. Con respecto a las reacciones de pirolisis, estas tienen lugar durante la segunda etapa, dando lugar a cambios químicos de los granos, resaltándose que grandes cantidades de $\mathrm{CO}_{2}$, agua y sustancias volátiles son liberadas y los granos se vuelven de color marrón, debido a la caramelización del azúcar acoplado a reacciones de Maillard. Donde se requiere disminución sustancial de la temperatura para evitar la quema del café (Rodrigues, Borges, Franca, Oliveira, y Correa, 2003).

Con base en lo expuesto es que el presente trabajo tiene como objetivo la evaluación de la relación entre las características clima, suelo y manejo del cultivo que pueden influir posteriormente en la calidad de taza y perfil, soportado en aspectos fundamentales como la identificación de las características del suelo, así como la evaluación de la relación entre prácticas de producción (precosecha, cosecha y poscosecha) en la producción del grano de los cafés especiales de la corporación cordillerana del municipio de Calarcá, departamento del Quindío.

\section{Metodología}

El presente estudio posee un carácter de enfoque de investigación empírico-analítico, soportado en la detección y análisis de variables asociadas a la producción de cafés especiales en fase de campo (suelos y agronómicos), e implica la comprensión de la dinámica 
de aquellas variables significativas que se convierten posteriormente en una serie de factores claves para el área bajo estudio, como en este caso la evaluación de la relación entre las características clima, suelo y manejo del cultivo de los cafés especiales de la Corporación Cordillerana del municipio de Calarcá.

Con respecto a las unidades experimentales, se trabajó una población de 19 fincas ubicadas en las veredas La Paloma y Santo Domingo, ubicadas en el municipio de Calarcá, del departamento del Quindío. El método de investigación y análisis estadístico se soportó en método de carácter correlacional con base en la relación existente entre variables, la cual puede ser positiva (a medida que aumenta $X i$ aumenta $Y i$ ) o negativa (a medida que aumenta $X i$ disminuye $Y i$ y viceversa), enfocado hacia el análisis de los factores agronómicos y medioambientales que pueden influir en la calidad de los cafés especiales, cuya fuente primaria de información es la base de datos aplicada a productores de la Corporación Cordillerana del Municipio de Calarcá.

Con el fin de relacionar los factores multivariantes que coadyuven a la formulación de estrategias tendientes a la dinámica del efecto de las condiciones de campo así como de beneficio del café sobre la calidad del mismo en taza, se lleva a cabo la técnica estadística del tipo análisis factorial de correspondencias múltiples, el cual es un sistema de análisis multivariante aplicado para evaluar la semejanza entre individuos con respecto a atributos analizados como variables y permite determinar además una tipología de tales individuos; también permite establecer que grupos de variables están correlacionadas (Lebart, Morineau \& Piron, 1995) y con base en valores test ( $t$-student) se identifican las variables de mayor significancia (confiabilidad del 95\%) al interior del estudio.
Asociado al análisis de correspondencias múltiples, se lleva a cabo un análisis de clasificación, por medio del análisis jerárquico de clústeres, con el fin de establecer grupos de interés que generan perfiles correlacionados que corroboran el análisis de correspondencias y para el presente estudio, dichas clasificaciones se realizan a través de distancias euclidianas, obteniéndose clasificaciones jerárquicas ascendentes por medio de dendogramas.

\section{Resultados y discusión}

Para el caso de la dinámica del suelo, se llevaron a cabo análisis factoriales de las condiciones del mismo como son $\mathrm{pH}$, concentraciones de fósforo, potasio, magnesio, aluminio y saturación de dicho elemento con el fin de determinar aquellas variables y factores significativos que determinan la dinámica del mismo.

Al realizar el análisis estadístico de histograma de valores propios, se detectaron dos planos factoriales que explican el 39,71\% de la información como se evidencia a continuación y con base en los valores test, se aprecia que a menor $\mathrm{pH}$, se evidencia una mayor saturación de aluminio y mayor concentración de materia orgánica. Es de agregar además que existe influencia significativa de las variedades de café castillo y caturra mezclado con castillo.El análisis factorial está asociado a una tendencia a la normalidad del $\mathrm{pH}$ del suelo, los respectivos altos contenidos de materia orgánica, bajas concentraciones de calcio y baja saturación de aluminio. Mientras que el segundo factor se asocia a $\mathrm{pH}$ ácidos, altos contenidos de materia orgánica, así como alta saturación de aluminio (ver figura 1)

Figura 1. Plano factorial de análisis multivariado general para condiciones del suelo

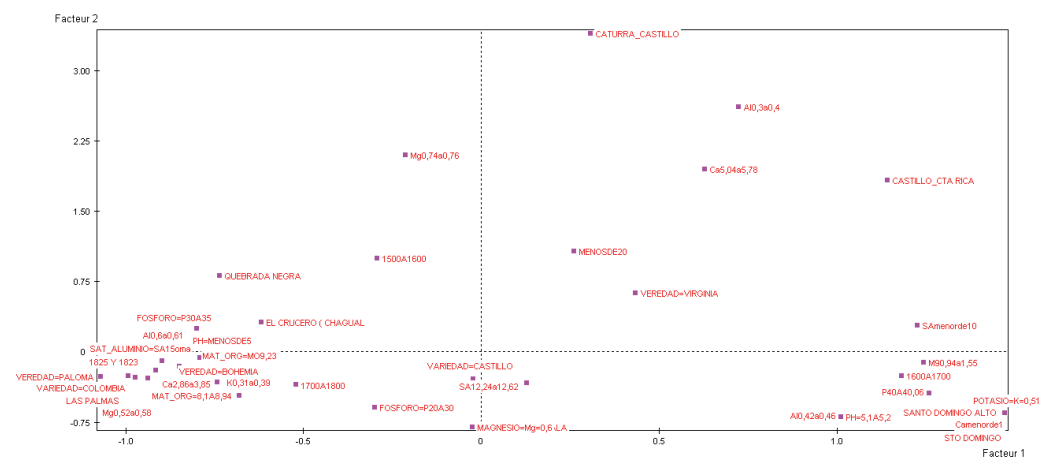

Fuente los autores 
Complementario al respectivo análisis, se llevaron a cabo análisis de clústeres, detectándose dos tipologías de condiciones de suelo, lo cual se puede apreciar en el respectivo dendograma (ver figura 2).

Figura 2. Dendograma general para condiciones del suelo

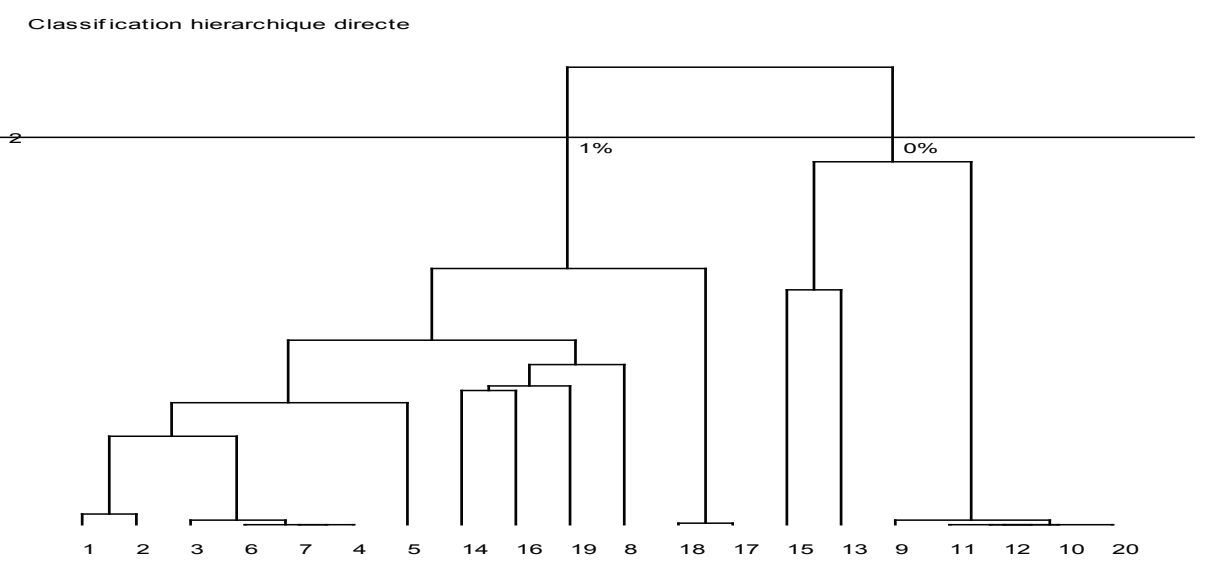

Fuente los autores

A partir de la conformación de grupos encontrada en el dendograma, se generaron los dos clúster según el tipo de condiciones del suelo (tablas 1 y 2):

Tabla 1. Clase 1 de fincas según características del suelo

\begin{tabular}{|c|c|c|c|c|c|c|c|c|c|}
\hline \multirow[t]{2}{*}{ V.TEST } & \multirow[t]{2}{*}{ PROBA } & \multicolumn{3}{|c|}{$\begin{array}{l}---- \text { POURCENTAGES }---- \\
\text { CLA/MOD MOD/CLA GLOBAL }\end{array}$} & $\begin{array}{l}\text { MODALITES } \\
\text { CARACTERISTIQUES }\end{array}$ & \multicolumn{2}{|l|}{ DES VARIABLES } & \multirow{2}{*}{$\begin{array}{c}\text { IDEN } \\
\text { aala }\end{array}$} & \multirow{2}{*}{$\begin{array}{r}\text { POIDS } \\
13\end{array}$} \\
\hline & & & & 65.00 & CLASSE $1 / 2$ & & & & \\
\hline 3.71 & 0.000 & 100.00 & 92.31 & 60.00 & $\mathrm{~K} 0,31 \mathrm{a} 0,39$ & POTASIO & {$[\mathrm{AF}]$} & $\mathrm{AF}_{-} 1$ & 12 \\
\hline 3.71 & 0.000 & 100.00 & 92.31 & 60.00 & $\mathrm{Ca} 2,86 \mathrm{a} 3,85$ & CALCIO & {$[\mathrm{AG}]$} & $\mathrm{AG}_{-} 1$ & 12 \\
\hline 3.31 & 0.000 & 100.00 & 84.62 & 55.00 & Alo, $6 \mathrm{a} 0,61$ & ALUMINIO & [AI] & AI_3 3 & 11 \\
\hline 2.96 & 0.002 & 100.00 & 76.92 & 50.00 & $\mathrm{PH}=\mathrm{MENOSDE} 5$ & PH & {$[\mathrm{AC}]$} & $\mathrm{AC}_{-}^{-} 4$ & 10 \\
\hline 2.96 & 0.002 & 100.00 & 76.92 & 50.00 & SAT_ALUMINIO=SA15oma & SAT_ALUMINIO & {$[\mathrm{AJ}]$} & $\mathrm{AJ}_{-}^{-} 2$ & 10 \\
\hline 2.96 & 0.002 & 100.00 & 76.92 & 50.00 & MAT_-ORG $=8,1 \mathrm{~A} 8,94$ & MAT_ORG & {$[\mathrm{AD}]$} & $\mathrm{AD}_{-}^{-}{ }^{3}$ & 10 \\
\hline 2.63 & 0.004 & 100.00 & 69.23 & 45.00 & $\mathrm{MgO}^{-}, 52 \mathrm{aO}, 58$ & MAGN̄ESIO & {$[\mathrm{AH}]$} & $\mathrm{AH}_{-}^{-} 2$ & 9 \\
\hline
\end{tabular}

Fuente los autores

La primer tipología representa el $65 \%$ de las fincas analizadas se caracterizan por pH menores de 5,0, altos niveles de saturación de aluminio, así como altos contenidos de materia orgánica en el suelo, sumado a niveles de calcio que fluctúan entre 2,86 y 3,85; 0,31 a 0,39 de potasio y 0,52 a 0,58 de magnesio, respectivamente.

Tabla 2. Clase 2 de fincas según características del suelo

\begin{tabular}{|c|c|c|c|c|c|c|c|c|c|}
\hline \multirow[t]{2}{*}{ V.TEST } & PROBA & \multicolumn{3}{|c|}{$\begin{array}{l}---- \text { POURCENTAGES ---- } \\
\text { CLA/MOD MOD/CLA GLOBAL }\end{array}$} & $\begin{array}{l}\text { MODALITES } \\
\text { CARACTERISTIQUES }\end{array}$ & \multicolumn{2}{|l|}{ DES VARIABLES } & IDEN & POIDS \\
\hline & & & & 35.00 & CLASSE $2 / 2$ & & & aa2a & 7 \\
\hline 4.21 & 0.000 & 100.00 & 100.00 & 35.00 & SAmenorde 10 & SAT_ALUMINIO & {$[\mathrm{AJ}]$} & $\mathrm{AJ}_{-}{ }^{3}$ & 7 \\
\hline 3.04 & 0.001 & 85.71 & 85.71 & 35.00 & $6,22 \mathrm{~A} 6,71$ & MAT_ORG & {$[\mathrm{AD}]$} & $\mathrm{AD}_{-}^{-1}$ & 7 \\
\hline 3.04 & 0.001 & 85.71 & 85.71 & 35.00 & M90,94a1, 55 & MAGN̄ESIO & {$[\mathrm{AH}]$} & $\mathrm{AH}_{-}^{-} 1$ & 7 \\
\hline 3.00 & 0.001 & 100.00 & 71.43 & 25.00 & POTASIO $=K=0,51$ & POTASIO & {$[\mathrm{AF}]$} & $\mathrm{AF}_{-}^{-} 3$ & 5 \\
\hline 3.00 & 0.001 & 100.00 & 71.43 & 25.00 & Camenorde 1 & CALCIO & [AG] & $\mathrm{AG}_{3}^{-}$ & 5 \\
\hline 2.45 & 0.007 & 83.33 & 71.43 & 30.00 & P $40 \mathrm{~A} 40,06$ & FOSEORO & {$[\mathrm{AE}]$} & $\mathrm{AE}_{4}^{-}$ & 6 \\
\hline 2.45 & 0.007 & 100.00 & 57.14 & 20.00 & STO DOMINGO & VEREDAD & [AA] & $\operatorname{AA} 10$ & 4 \\
\hline 2.45 & 0.007 & 83.33 & 71.43 & 30.00 & $\begin{array}{l}1600 \mathrm{~A} 1700 \\
1700\end{array}$ & ASNM & {$[A B]$} & $\mathrm{AB}_{2} 2$ & 6 \\
\hline
\end{tabular}

Fuente los autores 
Mientras la segunda tipología representa el $35 \%$ de los predios se caracterizan por niveles altos de (pero ligeramente inferiores al clúster 1) de materia orgánica, una menor saturación de aluminio, menores niveles de calcio, magnesio en concentraciones entre 0,94 a 1,55 ; potasio en concentración de 0,51 , fósforo en concentración de 40 a 40,06 y son representativos de estas condiciones los predios de las veredas Santo Domingo y la altitud entre 1600 y $1700 \mathrm{msnm}$.

Se generaron cuatro factores (ver figura 3) vinculados con las condiciones de las prácticas de producción, como son distancias entre plantas y surcos, periodicidad de la fertilización, existencia de sombrío en el cultivo, manejo poscosecha del grano en relación al tipo de maquinaria utilizada, número de veces de lavado, tiempo de fermentación y métodos de beneficio. El primer factor se asocia a la vereda Puerto Rico: la influencia de la altitud y las variedades caturra y castillo, plantas que pronto inician producción (24 meses) o en comienzo de producción (48 meses), sembrados a la sombra, sin reporte de manejo de fertilización, con distancia entre plantas de 120 a $180 \mathrm{~cm}$ y distancia entre surcos entre 140 y $180 \mathrm{~cm}$. Además, se toma en cuenta fincas que benefician principalmente con despulpadoras manual o tradicional y algunas usan baldes como medio para fermentar con diferentes tiempos de fermentación y secado en elba o al sol.

El segundo factor está representado por la vereda Las Palmas: cultivos con base en semisombra o al sol; no reportan fertilización, con plantas sembradas a distancias de 120 a $180 \mathrm{~cm}$ entre plantas y de 140 a $180 \mathrm{~cm}$ entre surcos, despulpan manualmente y fermentación del grano en baldes.

El tercer factor está representado por la vereda La Paloma: con cultivos de café variedades Castillo y Colombia, distancia entre plantas de $170 \mathrm{~cm}$, despulpadora mecánica 3 chorros y lavan una vez en tanque de fermentación.

El cuarto factor está caracterizado por las veredas La Bohemia y La Virginia: trabajan con despulpadora Jotagallo y mecánica a 3 chorros, utilizan tanque de fermentación y lavan 2 veces el grano.

Figura 3. Plano factorial de análisis multivariado general para prácticas de producción

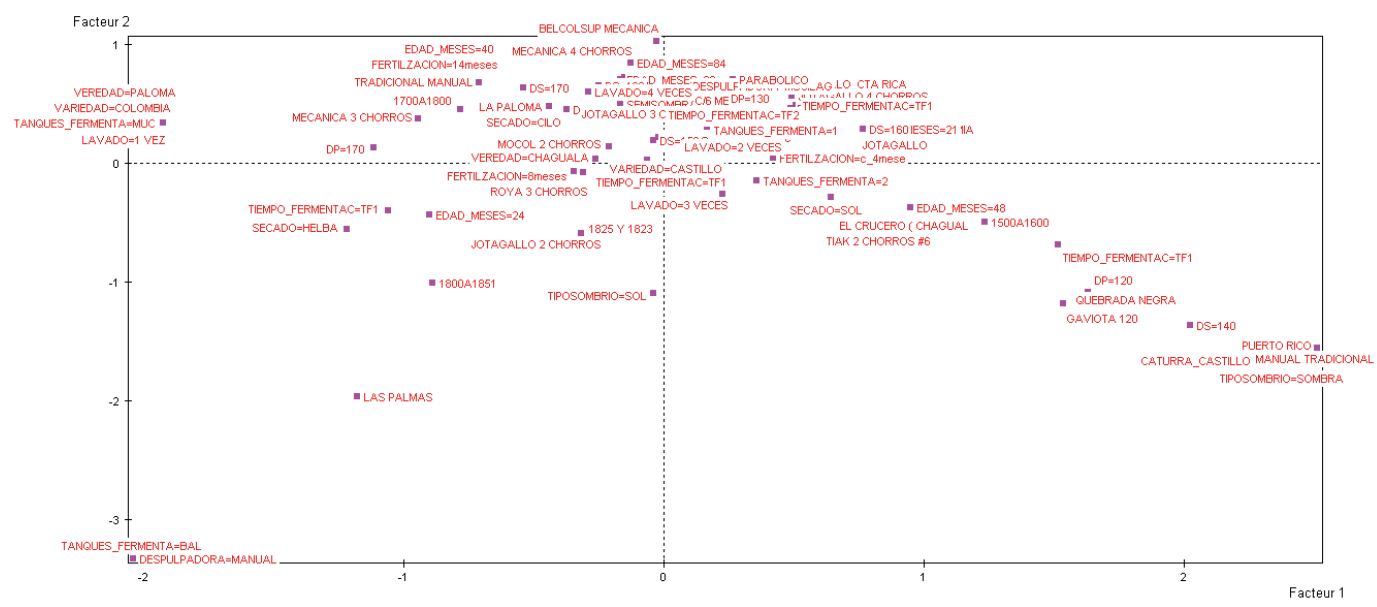

Fuente los autores

No obstante, al abordar el centroide (centro de la figura de plano factorial), se aprecia que la variedad castillo y el lavado del café por dos a tres veces son los aspectos más frecuentes de los productores, siendo las fincas de las veredas de Chagualá los más representativos de dichas prácticas (figura 4). 
Figura 4. Plano factorial de centroide en análisis multivariado general para prácticas de producción.

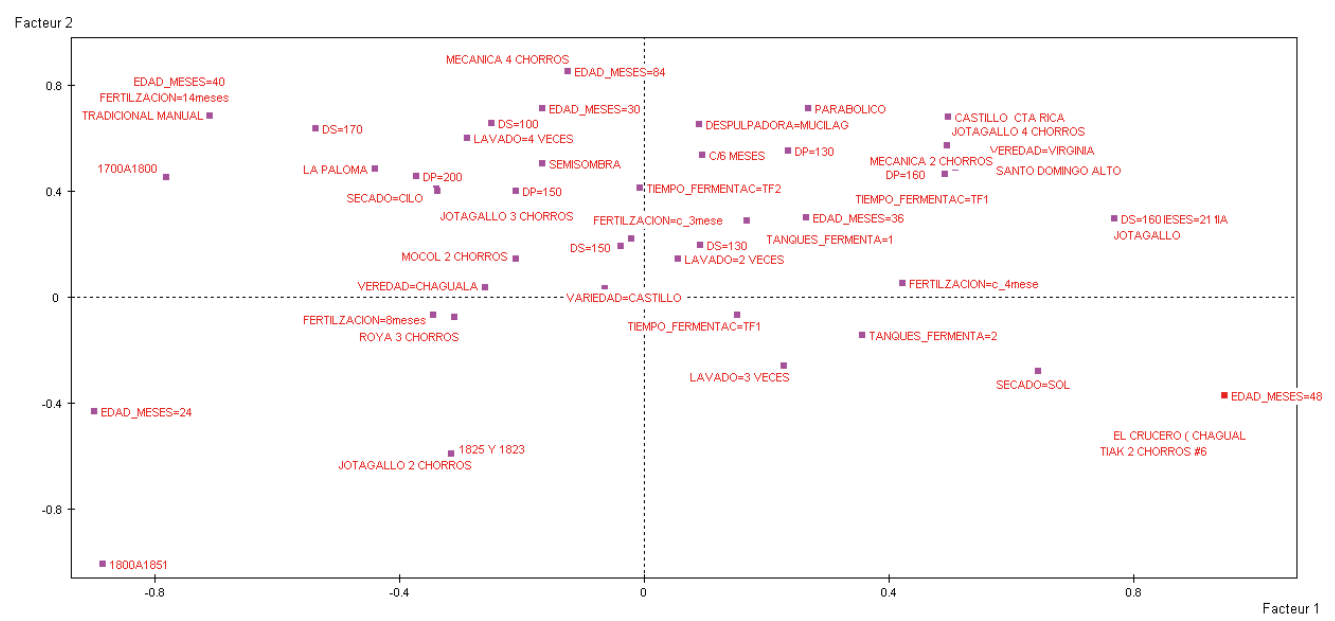

Fuente los autores

Complementario al respectivo análisis, se llevaron a cabo análisis de clústeres, detectándose dos tipos de condiciones de prácticas de producción lo cual se puede apreciar en el respectivo dendograma (Figura 5):

Figura 5. Dendograma general para prácticas de producción

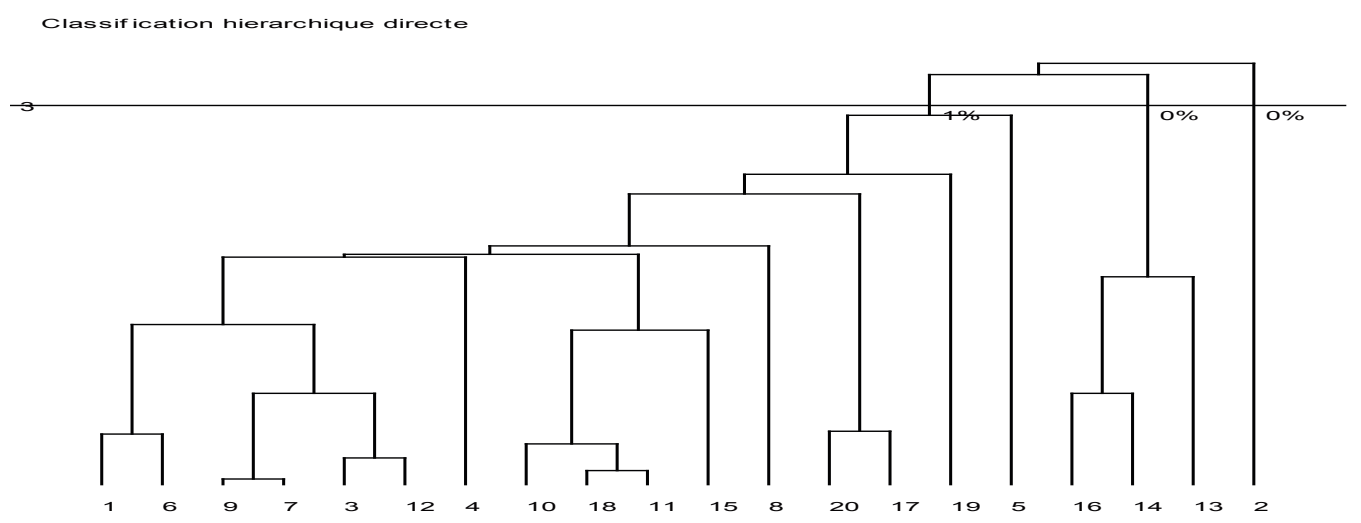

Fuente los Autores

Siendo los clústers los siguientes (Tablas 3 y 4): 
Tabla 3. Clase 1 de fincas según prácticas de producción

\begin{tabular}{|c|c|c|c|c|c|c|c|c|c|}
\hline V.TEST & PROBA & $\begin{array}{l}---- \text { POU } \\
\text { CLA/MOD }\end{array}$ & $\begin{array}{l}\text { CENTAGE } \\
\text { DD/CLA }\end{array}$ & GL ---- & $\begin{array}{l}\text { MODALITES } \\
\text { CARACTERISTIQUES }\end{array}$ & DES VARIABLES & & IDEN & POIDS \\
\hline & & & & 80.00 & CLASSE $1 / 3$ & & & aa1a & 16 \\
\hline 2.74 & 0.003 & 100.00 & 87.50 & 70.00 & SEMISOMBRA & TIPOSOMBRIO & [AM] & AM_1 & 14 \\
\hline
\end{tabular}

Fuente los autores

La primer tipología en función de aspectos agronómicos representa el 80\% de los cultivos manejan semisombra como práctica significativa de carácter agronómico.

Tabla 4. Clase 2 de fincas según prácticas de producción

\begin{tabular}{|c|c|c|c|c|c|c|c|c|c|}
\hline V.TEST & PROBA & $\begin{array}{l}--- \text { POC } \\
\text { CLA/MOD }\end{array}$ & $\begin{array}{l}\text { RCENTAGE } \\
\text { MOD/CLA }\end{array}$ & GL ---- & $\begin{array}{l}\text { MODALITES } \\
\text { CARACTERISTIQUES }\end{array}$ & DES VARIABLES & & IDEN & POIDS \\
\hline & & & & 15.00 & CLASSE $2 / 3$ & & & aa2a & 3 \\
\hline $\begin{array}{l}3.13 \\
2.38\end{array}$ & $\begin{array}{l}0.001 \\
0.009\end{array}$ & $\begin{array}{r}100.00 \\
60.00\end{array}$ & $\begin{array}{l}100.00 \\
100.00\end{array}$ & $\begin{array}{l}15.00 \\
25.00\end{array}$ & $\begin{array}{l}\mathrm{DP}=120 \\
1500 \mathrm{~A} 1600\end{array}$ & $\begin{array}{l}\text { DP } \\
\text { ASNM }\end{array}$ & $\begin{array}{l}{[\mathrm{AO}]} \\
{[\mathrm{AB}]}\end{array}$ & $\begin{array}{l}\mathrm{AO}{ }_{\mathrm{AB}}^{-1} \\
\mathrm{AB}\end{array}$ & $\begin{array}{l}3 \\
5\end{array}$ \\
\hline
\end{tabular}

Fuente los autores

Mientras que la segunda tipología se caracteriza por el 20 $\%$ de los predios giran en torno a distancia entre plantas de $120 \mathrm{~cm}$ y altitud de 1500 a 1600 msnm, siendo el único aspecto de manejo la distancia entre plantas como aspecto a tener en cuenta al momento de modela el cultivo.

Lo anterior permite denotar la capacidad de los productores de cafés especiales de la corporación cordilleranos con respecto a una responsabilidad ejercida en función de la sostenibilidad, corroborando lo planteado por Binder et al (2009) y Lancker y Nijkamp (2000), quienes afirman que la producción agrícola puede enfocarse de manera sostenible y que, a su vez, es parte de la cotidianidad de la población que por convicción la aplican como se aprecia en los resultados anteriores, reafirmando además lo planteado por Lundin (2003) y Berke y Manta (1999), que es posible la promoción y fortalecimiento de los sectores agrícolas siempre y cuando haya las condiciones para su fortalecimiento como es en el presente caso con respecto a los productores de cafés especiales de la Corporación cordillerana del Municipio de Calarcá-Quindío.

Finalmente, se evidencia en las tipologías de los clústeres establecidos, la identificación de indicadores que propenden por la producción sostenible; corroborando así lo planteado por Prescott y Allen (1995), Rajesh (2008) y Epstein (2009), respectivamente.

\section{Conclusiones}

Se aprecia que el $65 \%$ de las fincas analizadas se caracterizan por $\mathrm{pH}$ menores de 5,0, altos niveles de saturación de aluminio, así como altos contenidos de materia orgánica en el suelo, sumado a niveles de calcio que fluctúan entre 2,86 y 3,85 meq; 0,31 a 0,39 meq de potasio y 0,52 a $0,58 \mathrm{meq}$ de magnesio. Sin embargo, el $35 \%$ de las fincas se caracterizan por niveles altos de materia orgánica, una menor saturación de aluminio, menores niveles de calcio, magnesio en concentraciones entre 0,94 a 1,55 meq; potasio en concentración de 0,51 meq, fósforo en concentración de 40 a 40,06 meq y son representativos de estas condiciones los predios de la vereda Santo Domingo y la altitud entre 1600 y 1700 msnm.

Lo anterior hace denotar que las características propias de suelo de dicha región del departamento del Quindío; aunque es de resaltar altos niveles de materia orgánica, lo cual favorece, desde una perspectiva sostenible, la producción de café en dicha región.

Desde el contexto agronómico, se aprecia que los cafetales son jóvenes y en plena capacidad productiva (edad promedio de 39,5 meses), prepondera la variable castillo en los predios y el establecimiento de los cultivos 
bajo semisombra. Además, las plantaciones reportan fertilizaciones principalmente cada 4 meses en cultivos sembrados a 1,5 metros entre plantas y 1,5 metros entre surcos principalmente.

Desde el contexto multivariante, se aprecia que a menor $\mathrm{pH}$, se evidencia una mayor saturación de aluminio y mayor concentración de materia orgánica y es de resaltar que las veredas de referencia para la dinámica del suelo son La Paloma y Santo Domingo. Además, se aprecia que existe una preponderación significativa de las variedades de café castillo y caturra mezclado con castillo.

Con respecto a las prácticas de beneficio del grano, se aprecia que la máquina más utilizada es la del tipo 3 chorros, seguido de las máquinas Jota gallo 3 chorros y 4 chorros, así como la manual tradicional.

Es de agregar que los productores tienen la tendencia a utilizar un solo tanque para el beneficio del café y tienden a dejar fermentando el grano por 18 a 24 horas y lavarlo 3 veces. En cuanto al secado del mismo, se hace principalmente al sol, seguido del uso de elba y cilo.

Sumado a lo anterior, se resalta que el énfasis de los productores gira en torno a mantener los lotes en buen estado agronómico y fitosanitario, por tal motivo, el uso de semisombra en el cultivo se convierte en un aspecto determinante para dichos productores.

Desde el contexto multivariante, se aprecia que las altitudes y las características propias de cada vereda no influyen significativamente en la calidad de taza del café.

\section{Referencias bibliográficas}

Bansal, P; Roth, K (2000). Why companies go green: a model of ecological responsiveness, The Academy of Management Journal 43(4), 717-736.

Berke, P., Manta, M. (1999). Planning for Sustainable Development: Measuring Progress in Plans; Working Paper; Lincoln Institute of Land Policy. Cambridge, Massachusetts. http://www. lincolninst.edu/pubs/pubdetail.asp? $\mathrm{id}=58$

Binder, C.; Feola, G.; Steinberger, J (2009). Considering the normative, systemic and procedural dimensions in indicator-based sustainability assessments in agriculture. Environmental Impact Assessment Review, 30 71-81
Ebert, U., Welsch, H. (2004). Meaningful environmental indices: asocial choice approach. J. Environ. Econ. Manage. 47, 270-283.

Epstein, M (2009). Sostenibilidad Empresarial: Administración y Medición de los Impactos Sociales, Ambientales y Económicos. Bogotá: EcoeEdiciones

Epstein, M; Westbrook, R (2001). Linking actions to profits in strategic decision making, MIT Sloan. Management Review Spring, 39-49.

Freeman, R (1984). Strategic Management: A Stakeholder Approach. Boston:Pitman

Holmberg, J., Karlsson, S. (1992). On Designing Socio-ecological Indicators. In: Svedin, U., Anianssons, B. (Eds.), Society and the Environment: A Swedish Research Perspective. Kluwer Academic Publishers, Dordrecht, Netherlands, pp.89-106.

Lancker, E., Nijkamp, P. (2000). A policy scenario analysis of sustainable agricultural development options: a case study for Nepal. Impact Assess. Project Appraisal 18 (2), 111-124.

Lebart, L; Morineau, A; Piron, M (1995).Statistique exploratoire multidimensionnelle (2a Ed). Paris: Dunod.

Lundin, U. (2003). Indicators for Measuring the Sustainability of Urban Water Systems - a Life Cycle Approach, PhD Thesis, Department of Environmental Systems Analysis, Chalmers University of Technology, Go“ teborg, Sweden.

Lozano, A; Yoshida, P. (2008). Índice de Competitividad Regional Cafetero. En: http://www. federaciondecafeteros.org/static/files/Indice competitividad cafetero.pdf, acceso en agosto de 2015.

Ness, B., Urbel_Piirsalu, E., Anderberg, S., Olsson, L. (2007).Categorising tools for sustainability assessment. Ecol. Econ.60, 498-508.

Rajesh, S; Murty, H.R; Gupta, S,K; Dikshit, A.K (2008). An overview of sustainability assessment methodologies. Bhilai Steel Plant, SAIL, Bhilai \& Centre for Environmental Science and 
Engineering, Indian Institute of Technology, Mumbai.

Rodrigues, M. A. A., Borges, M. L. A., Franca, A. S., Oliveira, L. S. \& Correa, P. C. (2003). Evaluation of physical properties of coffee during roasting. Agricultural Engineering International: The CIGR. Journal of Scientific Research and Development, 5, 12.

Schmidt-Bleek (1994). How to reach a sustainable economy. Wuppertal Papers 24, 10-13.

Wackernagel, M., Rees, W. (1996). Our Ecological Footprint: Reducing Human Impact on the Earth. BC, Gabriola Island:New SocietyPublishers, 